Purdue University

Purdue e-Pubs

Predicting the Growth of Many Droplets During Vapor-DiffusionDriven Dropwise Condensation Experiments Using the Point Sink Superposition Method

J. Castillo

Purdue University

J. A. Weibel

Purdue University, jaweibel@purdue.edu

Follow this and additional works at: https://docs.lib.purdue.edu/coolingpubs

Castillo, J. and Weibel, J. A., "Predicting the Growth of Many Droplets During Vapor-Diffusion-Driven Dropwise Condensation Experiments Using the Point Sink Superposition Method" (2019). CTRC Research Publications. Paper 361.

http://dx.doi.org/https://doi.org/10.1016/j.ijheatmasstransfer.2018.12.068

This document has been made available through Purdue e-Pubs, a service of the Purdue University Libraries.

Please contact epubs@purdue.edu for additional information. 


\title{
Predicting the growth of many droplets during vapor- diffusion-driven dropwise condensation experiments using the point sink superposition method
}

\author{
Julian E. Castillo and Justin A. Weibel ${ }^{*}$
}

\author{
School of Mechanical Engineering, Purdue University, West Lafayette IN 47907 USA. \\ ${ }^{*}$ Corresponding author. \\ E-mail addresses: casti119@purdue.edu (Julian E. Castillo), jaweibel@ purdue.edu (Justin A. Weibel)
}

\begin{abstract}
Water vapor present in humid air will condense in the form of many small droplets on a cooled substrate. After nucleation, the diffusion of vapor from the environment to the droplets dominates their growth by condensation, and therefore, all droplets must compete for the vapor available in the surroundings. Models that assume droplets grow in isolation or as an equivalent film poorly capture their interaction during vapor-diffusion-driven condensation and do not correspond with experimental condensation rates. By treating the droplets as point sinks, the interaction between all droplets in a system can be captured by superposing the vapor distributions of each droplet. This paper presents direct comparisons of condensation rates measured in experiments and predicted with a point sink superposition method. The results indicate that it is critical to consider a large number of interacting droplets to accurately predict the condensation behavior. Even though the intensity of the interaction between droplets decreases sharply with their separation distance, droplets located relatively far away from a given droplet must be considered to accurately predict its condensation rate, due to the large aggregate effect of all such far away droplets. By considering an appropriate number of interacting droplets in a system, the point sink superposition method is able to predict experimental condensation rates to within 5\%. Diffusion-based models that neglect the interactions of droplets located far away, or approximate droplet growth as an equivalent film, are shown to overpredict condensation rates.
\end{abstract}




\section{Nomenclature}

$B$ matrix containing the droplet locations and sizes

c vapor concentration

D diffusion coefficient

$L \quad$ characteristic length of the sample

$N \quad$ number of droplets in the system

$\dot{m} \quad$ condensation rate of individual droplet

$\dot{M} \quad$ total condensation rate

$\vec{r} \quad$ position vector

$R \quad$ single droplet radius

RH relative humidity

Pe Péclet number $(\mathrm{Pe}=\zeta U / D)$

S condensing area

$t \quad$ time

$T \quad$ temperature

$U \quad$ air velocity above the substrate

$(x, y, z) \quad$ Cartesian coordinates

\section{Greek}

$\beta \quad$ volumetric thermal expansion coefficient

$\delta \quad$ thickness of hydrodynamic boundary layer

$\zeta \quad$ thickness of the concentration boundary layer

$\eta \quad$ condensation correction factor 
$\theta \quad$ contact angle

$\sigma \quad$ density per unit area of the point sink

$\kappa \quad$ extension of the region that encloses a group of droplets

$\mu \quad$ power law exponent

$\Psi \quad$ intensity of droplet-to-droplet interaction

\section{Subscripts}

$c \quad$ contact area between the droplet and substrate

$\exp$ experiment

film film-like growth model

iso isolated from all neighboring droplets

$l \quad$ liquid

PSSM point sink superposition method

$s \quad$ at the surface of the drop

sub at the substrate

sys in the system of droplets

$\infty \quad$ far field

\subsection{Introduction}

In locations where access to fresh water is limited, water collection from humid air has the potential to be an alternative source [1,2]. The adoption of water harvesting is made challenging due to the limited amount of water vapor present in the air, limitations on the cooling power available to drive condensation [3], and variability of environmental conditions [1,3]. Recent efforts have focused on maximizing water yield and removal of condensate by engineering the chemistry and morphology of the substrate [5-8]; however, models that accurately describe 
condensation rates are lacking. Accurate prediction of water harvesting capabilities during dropwise condensation from humid air could lead to the improved design and scalability of water harvesting systems $[1,8]$.

When water vapor present in the air condenses in a dropwise manner, droplets grow by two mechanisms: vapor diffusion and droplet coalescence. The water vapor must diffuse from the environment to the surfaces of the droplets; each droplet must compete with its neighbors for the vapor available in the surroundings. As a result, the growth of each individual droplet depends on the local distribution of surrounding vapor. At constant substrate temperature and vapor concentration in the air, droplet sizes and their spatial distribution determine droplet growth characteristics. Dropwise condensation proceeds in a cyclical manner from droplet nucleation to ultimate roll-off, where the characteristic sizes and spatial distribution have been typically described according to three different stages of growth [9-11]. During stage (i), droplets nucleate at various locations on the substrate separated by distances that are relatively larger than the average radius of the droplets. A common assumption is that droplets grow with negligible interactions during this stage, as if they were isolated from each other. As droplets grow, the distance between their surfaces decreases, such that at later times during this stage $(i)$, droplets are often assumed to grow as an equivalent liquid film with a volume equal to the volume of all the droplets combined. In stage (ii), droplet coalescence occurs, which increases the average distance between droplets and broadens the size distribution (large droplets that merge with their neighbors coexist with small droplets that have not yet coalesced). The fraction of substrate coverage by the droplets have been observed to be constant and droplet growth exhibits self-similar characteristics in this stage. New droplets nucleate in the bare spaces between the droplets, creating two generations of droplets on the substrate: newly formed small droplets and large droplets that formed during the earlier stages of growth. Despite the various sizes of droplets on the substrate during regime (ii), droplet growth is commonly approximated by the film-like model. Finally, during stage (iii), droplets that reach the capillary length will roll off the substrate due to gravitational forces if the substrate has some inclination.

The isolated and film-like models are widely used in the literature to describe droplet growth, but there are some growth characteristics that these simplified descriptions cannot capture. For example, Ucar and Erbil [10] showed that the isolated growth model overpredicts experimental condensation rates of equally sized neighboring droplets growing in close proximity by up to $40 \%$. 
Castillo et al. [11] demonstrated in experiments that droplet growth due to vapor diffusion follows a power law with an exponent that is sensitive to relative humidity. Medici et al. [12] observed differences in growth rates that result from geometric discontinuities; droplets near the edges of a substrate grow $\sim 500 \%$ faster than a droplet near the center. These discrepancies in droplet growth were approximated with a modified version of the film-like model that accounted for the local variation of vapor distribution by tuning the mean water vapor concentration in the locality of each droplet. The local distribution of water vapor also controls the growth of new droplets between pre-existing on the substrate. Nath and Boreyko [13] used a scaling analysis to approximate the extension of dry zones around large droplets in which droplet growth is suppressed. The local vapor distribution has also been shown to play a fundamental role in understanding several freezing phenomena such as inter-droplet ice bridging, dry zones around frozen droplets, and frost halos $[13,14]$. It is evident that the interaction between droplets is critical to accurately describe the process of vapor-diffusion-driven condensation on hydrophobic substrates.

To account for the complete set of interactions between all droplets on the substrate, communicated through the vapor concentration field, we recently developed a point source superposition method to describe droplet growth during dropwise condensation [15]. By assuming that each droplet can be treated as point sink for vapor, this method solves for the condensation rate of each droplet within a system of many droplets by superposing a modified version of Popov's solution [16] for the condensation of individual droplets; the point sink superposition method was demonstrated to be in reasonable agreement with complete numerical solutions of the vapor diffusion equation.

In the current work, we directly compare droplet condensation rates predicted by the point sink superposition method against condensation experiments, for dropwise condensation from humid air. Droplet growth is tracked during the condensation process in order to measure the condensation rate of each droplet, which is then compared to the instantaneous condensation rates predicted by the point sink superposition method. At the experimental conditions, model predictions of the instantaneous and temporal droplet condensation rates are investigated as function of the number of interacting droplets taken into account in the system. These droplet growth characteristics are discussed in terms of the interactions between each droplet and all of its neighbors during different stages of droplet growth. By incorporating the interaction of all the 
droplets, the point sink superposition method can be used as a tool to accurately predict droplet growth during dropwise condensation from humid air.

\subsection{Experimental description}

\subsection{Substrate preparation}

Smooth $20 \times 20 \mathrm{~mm}^{2}$ silicon substrates were silanized to render them hydrophobic. The silicon substrates were first cleaned with piranha solution $\left(3: 1 \mathrm{H}_{2} \mathrm{SO}_{4}: \mathrm{H}_{2} \mathrm{O}_{2}\right)$ for $10 \mathrm{~min}$, rinsed with DI water, and blown dry with $\mathrm{N}_{2}$. The samples were then spin-coated with a 1:1000 v/v solution of trichloro(1H,1H,2H,2H-perfluorooctyl) silane (Sigma-Aldrich) in hexane (Sigma-Aldrich) at 2500 rpm and baked on a hot plate at $140{ }^{\circ} \mathrm{C}$ for $1 \mathrm{~h}$ to evaporate the solvent. The sample surface had a measured average roughness of $0.001 \mu \mathrm{m}$ (NewView 6000, Zygo) and a contact angle with a $5 \mu \mathrm{l}$ droplet water of $119.5 \mathrm{deg}$, as measured from side-view images using a goniometer (ramé-hart, 290-F1).

\subsection{Experimental setup}

Condensation experiments were carried out inside a custom-made environmental chamber equipped with air conditioning and cooling systems. A description of the condensation test facility is presented here, which is a modified version of the facility previously introduced in detail in Ref. [11]. A schematic diagram showing the flow conditioning systems and a picture of the test section are provided in Figure 1. The humidity conditioning system mixes dry and humid air adiabatically to achieve the relative humidity desired in the downstream test section. Two mass flow controllers (FMQ 5400, 0-2 SLM, Omega) regulate the amount of dry gas that passes through the humidifier or bypasses to the adiabatic mixer, before the gas mixture enters the test section. A hygrometer (HX93V2-RP1, Omega) inside the test section measures the relative humidity and provides feedback to a PID control loop that actuates the two mass flow controllers. All signals are acquired using a data acquisition system (PCI-6120, National Instruments). The relative humidity can be controlled to set points between $20 \%$ and $75 \%$ with a measurement uncertainty of $\pm 2.5 \%$. The temperature inside the test section is monitored by a built-in temperature sensor in the hygrometer with a measurement uncertainty of $\pm 0.6^{\circ} \mathrm{C}$.

The temperature of the substrate is maintained constant through the experiments by using the cooling system attached to the test section as depicted in Figure 1. A chiller (ThermoFlex, 900 W, 
Thermo Scientific) circulates cooled water through a cooling plate (CP12, Lytron) that mounts flush with bottom of the test section and maintained at a constant temperature. A thermoelectric cooler (ZT8, Laird Technologies) attached to the top of the cold plate is used for fine-tuning of the sample temperature.

The stack of components inside the test section used to thermally bridge the sample to the cooling system was modified as compared to Ref. [11]. An aluminum block, placed between the thermoelectric device and sample substrate, was decreased in size $\left(20 \times 20 \times 6.35 \mathrm{~mm}^{3}\right)$ to reduce thermal inertia of the system and improve the response time of the sample temperature control system. The temperature of the sample was sensed by a 2 wire Pt1000 RTD (PRTF-10-2-100-1/412-E-GG, Omega) that fits in a hole located at $2.5 \mathrm{~mm}$ from the top surface of the aluminum sample holder. The temperature signal closes a PID control loop programmed in a temperature controller (MTTC-1410, Laird Technologies). The substrate is attached to the sample holder with carbon conductive double-sided tape (PELCO Image Tabs). The cooling system is capable of maintaining a constant set-point temperature to within $\pm 1.0{ }^{\circ} \mathrm{C}$ and with a measurement uncertainty of $\pm 0.1^{\circ} \mathrm{C}$ as sensed by the RTD.

\subsection{Experimental procedure and visualization}

The substrate is attached to the sample holder, which is then attached to the thermoelectric cooler inside the test section. The test section is sealed and purged with dry air for $\sim 20$ min until the relative humidity reaches $20 \%$. At the same time, the cold plate is used to cool down the entire test section until the temperature of the sample and the gas mixture inside the chamber stabilize at $\sim 20^{\circ} \mathrm{C}$.

Once the gas inside the chamber has reached constant temperature and relative humidity, the gas mixture flow controller is turned on. Once the relative humidity inside the chamber reaches the set point, the thermoelectric cooler controller is turned on and set to the desired sample temperature. After $\sim 1 \mathrm{~min}$, the temperature of the sample reaches a constant value within $\pm 2 \%$ of the set-point temperature. The relative humidity controller compensates for drying of the air inside the chamber caused by the rapid sample cooling. Within $\sim 2$ min after the thermoelectric has been turned on, the relative humidity inside the chamber reaches a constant value with $\pm 5 \%$ error relative to the set-point value.

Each condensation experiment is carried out at a specified relative humidity and substrate temperature. In this paper, condensation experiments are performed at a constant substrate 
temperature of $T_{\text {sub }}=10.0^{\circ} \mathrm{C}$, constant air temperature of $T_{\infty}=20.5^{\circ} \mathrm{C}$, and three different relative humidity values of $R H=70,60$, and $50 \%$.

Video of the condensation process is recorded viewing normal to the sample using a camera (EO-5023M, Edmund Optics) at 150× magnification using a long-focal-distance zoom lens (VHZ50L, Keyence) with in-line illumination provided by a $300 \mathrm{~W}$ Xe arc lamp (Titan 300, Sunoptic Technologies). The video recording was synchronized with the data acquisition system, such that each image corresponds to a known set of experimental conditions. Once the sample temperature and the chamber relative humidity are constant, the camera starts recording, corresponding with the initial time $t=0 \mathrm{~min}$.

\subsection{Image post-processing}

An automated video post-processing script developed in MATLAB is used to obtain information about the spatial distribution and temporal evolution of the droplets during the condensation experiments. The algorithm extracts a sequence of snapshot images from the video recordings and, within each image, detects each droplet by recognizing their boundaries as circles with centers coinciding with the centers of the droplets. Figure 2 (a) and (b) show snapshot images taken at 9 and 16 min during experiment at $R H=70 \%$. The edges of the droplets are depicted as blue circles, while the dashed red lines represent the Delaunay triangulation from the droplet centers.

The spatial resolution was calibrated by measuring circular patterns on a calibration target $\left(100 \times 100 \mathrm{~mm}^{2}, 1.0 \mathrm{~mm}\right.$ spacing, Glass Distortion Target, Edmund Optics $)$ using the detection algorithm described above, which is capable of detecting subpixel variations in circular patterns. At $150 \times$ magnification, the calibrated spatial resolution is $0.88 \pm 0.0015 \mu \mathrm{m} /$ pixel. This apparently high sensitivity comes from the ability of the circle detection algorithm capable of detecting subpixel variations in circular patterns. The $\sim 1.7 \times 1.7 \mathrm{~mm}^{2}$ field of view surveyed during the experiments is located near the center of the $20 \times 20 \mathrm{~mm}^{2}$ substrate. Droplets with contact radii from $12 \mu \mathrm{m}$ to $100 \mu \mathrm{m}$ were detected. The droplets are assumed to be spherical caps with a constant contact angle equal to 119.5 degrees.

The algorithm is used to track individual droplets, as is demonstrated for droplet $a 1$, which appears in both sequential frames of Figure 2. The algorithm is also used to track groups of droplets among which coalescence events can be identified as time progresses. For example, the 
nearest neighbors of droplet $a 1$, droplets $b 1$ through $b 7$ in Figure 2 (a), correspond to droplets $c 1$ through $c 5$ in Figure 2 (b), where droplets $b 1$ and $b 2$ merged to form droplet $c 2$.

The condensation rate of each droplet is calculated as the ratio between the change in mass over the time elapsed between successive images, using a five-point moving average to filter spurious noise. Information regarding coalescence events is used to note changes in condensation rate due to coalescence. Changes in the condensation rates of individual droplets between $\sim 10^{-15}$ to $\sim 10^{-13} \mathrm{~kg} / \mathrm{s}$ can be resolved by the algorithm, with uncertainties proportional to the measurement uncertainty in droplet size.

Because the area of the substrate that can be surveyed at high magnification is limited to a region that is only $\sim 8.5 \%$ of the total substrate area, an algorithm was developed to generate artificial droplets outside the interrogation window. The algorithm creates droplets to mimic the size distribution and density of the droplets inside the interrogation window. The purpose and use of this algorithm are further described in the results (Sections 4.1.1 and 4.1.2).

\subsection{The point sink superposition method (PSSM)}

The point sink superposition method solves for the diffusion of vapor within the air domain surrounding the droplets during dropwise condensation. Our previous work [15] provides a complete description of the method which is briefly summarized here. The model assumes: (i) droplets can be treated as point sinks located at the droplet centers, (ii) the condensation process can be treated as quasi-steady state, (iii) there is negligible thermal resistance across the droplets (i.e., the temperature at the surface of the droplet is equal to the temperature of the substrate), (iv) the contact angle of the droplets is independent of droplet size, and (v) changes in vapor concentration at the surface of the droplets due to curvature are negligible. Under these assumptions, the vapor concentration field around the droplets $c(\vec{r})$ is given by the Poisson equation:

$$
\nabla^{2} c(\vec{r})=\sigma \delta\left(\vec{r}-\vec{r}_{j}\right)
$$

where $\vec{r}$ describes any location in the domain, $\vec{r}_{j}$ is the location of the $j^{\text {th }}$ droplet in the domain with $j=1,2, \ldots, N$ and $N$ being the number of droplets, $\delta\left(\vec{r}-\vec{r}_{j}\right)$ is the Dirac delta function representing a point sink located at $\vec{r}=\vec{r}_{j}$, and $\sigma$ is the density per unit area of the point sink. The 
solution to equation (1) must satisfy the boundary conditions at the surface of the droplets and far away from the droplets. From assumptions (iii), (iv), and (v), the vapor concentration at the surface of all the droplets $c\left(\vec{r}_{j}+\vec{r}_{s j}\right)=c_{s}$ is calculated using the saturated vapor pressure at the temperature of the substrate. As discussed in Ref. [15], these assumptions are valid when there is a small temperature drop across the height of the droplet and additional effects of external convection, thermocapillary flows, and release of latent heat on the interface of the droplets are not considered. In the far field, the vapor concentration $c(\vec{r} \rightarrow \infty)=c_{\infty}$ corresponds to the vapor pressure at the temperature and relative humidity of the bulk air. The solution to equation (1) provides the concentration field within the air domain from which the condensation rate of individual droplets $\dot{m}_{s y s}$ can be calculated. Ref. [15] provides a closed-form solution for equation (1) which is reproduced here:

$$
\eta_{i}+\sum_{j=1, j \neq i}^{N} \eta_{j}\left(\frac{R_{c j}}{\left|\vec{r}_{i}-\vec{r}_{j}\right|}\right)=1,
$$

where $R_{c j}$ is the contact radius of the droplet, $\left|\vec{r}_{i}-\vec{r}_{j}\right|$ is the distance between droplet centers, $\eta$ is the condensation correction factor which is defined as the ratio between the condensation rate of the droplet within the system $\dot{m}_{s y s}$ and the isolated condensation rate $\dot{m}_{i s o}$. Therefore $\eta$ takes a value between unity (isolated droplet growth) and zero (droplet growth entirely suppressed due to surrounding droplets). We derive $\dot{m}_{i s o}$ by modifying Popov's evaporation model [16] as:

$$
\dot{m}_{i s o}=\pi R_{c} D\left(c_{\infty}-c_{s}\right) f(\theta),
$$

where $f(\theta)$ is an function of the contact angle of the droplets $\theta$ (equation (8) in Ref [15]). Equation (2) can be rewritten in the matrix form as:

$$
\dot{m}_{s y s, i}=\dot{m}_{i s o, i} B_{i j}^{-1},
$$

where $B_{i j}$ is an $N$ by $N$ matrix with diagonal elements $(i=j)$ equal to unity and off-diagonal elements $(i \neq j)$ equal to:

$$
B_{i j}=\left(\frac{R_{c j}}{\left|\vec{r}_{i}-\vec{r}_{j}\right|}\right),
$$


The strength interaction between droplets depends on the distance between the droplets and their sizes. For an arbitrary droplet $i$, the intensity of the interaction with another droplet $j$ in the domain will decreases with the distance that separates them. Equation (4) provides a simplified way to calculate the condensation rate of all individual droplets within a system of many droplets on a substrate, while accounting for vapor distribution field, based only on thermodynamic variables (substrate temperature, air temperature, and relative humidity) and the geometry of the droplets (droplet sizes, contact angle, and locations).

\subsection{Results}

\subsection{Comparison of the point sink superposition method (PSSM) with experiments}

This section compares the instantaneous condensation rates of individual and groups of droplets measured during condensation experiments against predictions of the point sink superposition method (PSSM). The case considered in this section is for droplets growing during at $T_{\text {sub }}=10{ }^{\circ} \mathrm{C}, R H=70 \%$, and $T_{\infty}=20.5^{\circ} \mathrm{C}$. At these conditions, the water vapor concentration at the surface of the droplet is $c_{s}=0.0095 \mathrm{~kg} / \mathrm{m}^{3}$ and the vapor concentration in the far field is $c_{\infty}$ $=0.0126 \mathrm{~kg} / \mathrm{m}^{3}$. The PSSM calculation considers the size and location of the droplets inside the interrogation window as described in Section 2.4. Additional comparisons for experiments at $R H$ $=50 \%$ and $R H=60 \%$ are provided in the Supplementary Materials.

\subsubsection{Condensation rate of a single droplet}

A top-down view of the model domain employed to predict the condensation rate of a single droplet is depicted in Figure 3 (a). Near the center of the image, the droplets inside the field of view during the experiment at $t=31 \mathrm{~min}$ are filled red, while the droplets outside the camera field of view are filled white. Among the droplets inside the field of view, the condensation rate is predicted with the PSSM for the droplet of interest (filled blue). The instantaneous condensation rate predicted for a droplet highly depends on the number of neighboring droplets considered in the calculation $\left(N_{P S S M}\right)$, due to the competition for surrounding vapor. The calculated condensation rate of the droplet of interest $\left(\dot{m}_{P S S M}\right)$ is plotted against $N_{P S S M}$ in Figure $3(\mathrm{~b})$, with $N_{P S S M}$ sorted in ascending order based on the distance from the droplet of interest. By increasing 
$N_{P S S M}$ in each calculation, the predicted $\dot{m}_{P S S M}$ converges to the measured condensation rate for the droplets of interest $\dot{m}_{\text {exp }}=1.77 \times 10^{-12} \pm 6.1 \times 10^{-13} \mathrm{~kg} / \mathrm{s}$ (represented by the horizontal line in Figure 3 (a)). This is quantified via the decrease in percentage error of $\dot{m}_{P S S M}$ relative to $\dot{m}_{\text {exp }}$ with increasing $N_{P S S M}$ and, similarly the decrease in the residual of $\dot{m}_{P S S M}$ between increments of $N_{P S S M}$ , as shown in Figure 3 (c)

When only the group of seven nearest neighbors to the droplet of interest is considered, connected by a green line in Figure 3 (a), the prediction is approximately eight times larger than the measured condensation rate. Vapor-diffusion-based models that only account for the interaction with nearest neighboring droplets would grossly overestimate the condensation rate of the droplet. As more droplets inside the field of view are added to the calculation, the relative error between $\dot{m}_{P S S M}$ and $\dot{m}_{e x p}$ drops sharply. However, after including all the red-filled droplets in the field of view $\left(N_{P S S M}=61\right), \dot{m}_{P S S M}$ is still a couple of times greater than $\dot{m}_{\text {exp }}$, which indicates that droplets located even outside the field of view have a significant effect on the vapor distribution surrounding the droplet of interest. Since the droplets located outside the field of view can not be surveyed, their effects are represented by creating artificial droplets in the domain, surrounding the field of view, using the algorithm described in Section 2.4. As more of these additional droplets are included in the calculation, $\dot{m}_{P S S M}$ continues to approach toward $\dot{m}_{\text {exp }}$. When $N_{P S S M}=564$ (purple vertical lines in Figure 3 (b) and (c)), the residual of the condensation rate is three orders of magnitude smaller than $\dot{m}_{\text {exp }}$ and of the same order of magnitude as the minimum condensation rate that can be detected by the experimental apparatus $\left(\sim 10^{-15}\right)$; at this point, the relative error in the prediction is $15.33 \%$. At $N_{P S S M}=753$, the relative error between $\dot{m}_{P S S M}$ and $\dot{m}_{\text {exp }}$ is $0.06 \%$.

From these results, we observed that a large number of droplets must be considered in a system to accurately predict the condensation rate. If a sufficient number of droplets are considered, the PSSM model can be used to accurately predict the condensation rate of a single droplet within the system. In general, the number of droplets required is expected to depend on the size and spatial distribution of its neighboring droplets. 


\subsubsection{Condensation rate of a group of droplets}

In this section, the condensation rate prediction is extended to a group of 30 droplets within the same domain as was considered in Section 4.1.1. A top-down view of the same domain shown in Figure 3 (a) is reproduced in Figure 4 (a). All droplets in the group of interest are filled blue inside the boundary defined by the yellow line; droplets in the experimental field of view are filled red. Figure 4 (b) shows the predicted total condensation rate of the group of droplets ( $\dot{M}_{\text {PSSM }}$ ) versus the number of droplets included in the prediction $\left(N_{P S S M}\right)$, where the blue horizontal line is the total condensation rate measured during experiments $\dot{M}_{\text {exp }}=3.04 \times 10^{-11} \pm 1.61 \times 10^{-12} \mathrm{~kg} / \mathrm{s}$. Each droplet of interest has been assigned an identification number shown in the zoomed-in view of the domain in Figure 5 (a). The condensation rate for each droplet within the group is shown in Figure 5 (b) for different values of $N_{P S S M}$.

When the prediction includes all droplets in the field of view $\left(N_{P S S M}=61\right), \dot{M}_{P S S M}$ is about three times larger than $\dot{M}_{\text {exp }}$, and the condensation rate of each individual droplet is also overpredicted, as shown in Figure 5 (b). As more droplets are included in the model, $\dot{M}_{\text {PSSM }}$ converges to the measured $\dot{M}_{\text {exp }}$, as indicated in Figure 4 (b). The residual of the calculated $\dot{M}_{\text {PSSM }}$ and the error relative to $\dot{M}_{\text {exp }}$ are plotted as a function of $N_{P S S M}$ Figure 4 (c); both quantities decrease as $N_{P S S M}$ increases. When $N_{P S S M}=526$, the difference between $\dot{M}_{P S S M}$ and $\dot{M}_{\text {exp }}$ reaches a minimum, and $\dot{m}_{P S S M}$ for each droplet approximates its corresponding $\dot{m}_{\text {exp }}$ value as shown in Figure 4 (b) and Figure 5 (b), respectively. In average, the relative error for individual droplets was $2.9 \%$ and standard deviation of $42.1 \%$. When $N_{P S S M}=569$, the residual of $\dot{M}_{P S S M}$ is three orders of magnitude smaller than $\dot{M}_{\text {exp }}$ and the model slightly underpredicts $\dot{M}_{\text {exp }}$ with an error of $-1.24 \%$.

The number of droplets that must be included in the domain to accurately predict the total condensation rate of groups of droplets is different at differing times of the condensation process, as the size and distribution characteristics of the droplets changes. Table 1 summarizes the number of droplets $N_{P S S M}$ required in the system to minimize the error in the condensation rate between the model and measurements, as well as the maximum cord length of the region that encloses these 
droplets $(\kappa)$. The $N_{P S S M}$ required to minimize the relative error decreases as the condensation process progresses, but $\kappa$ does not vary significantly, with an average equal to $2530 \pm 8 \mu \mathrm{m}$ for all the values reported in Table 1. During regime ( $i)$, the number of droplets per-unit-area is large; as droplet coalesce and grow during regime (ii), the number of droplets decreases. As a result, for a fixed area on the substrate, the number of droplets per unit area will reduce with time, as can be observed in the sequence of top-down view images of the domain shown in the Supplemental Materials for each time in Table 1. The value of $\kappa$ provides a case-specific criterion for assessing the number of droplets that must be included in a system to accurate predict the condensation rate.

\subsubsection{Temporal evolution of the condensation rate of single droplets}

This section focuses on predicting the temporal evolution of the condensation rate of individual droplets observed during the condensation experiments. Figure 6 (a) shows two typical droplets tagged as $d$ and $e$ in a sequence of images at $t=14.5$ and $17.0 \mathrm{~min}$. The measured and predicted time-varying condensation rates of droplets $d$ and $e$ are plotted in Figure 6 (b). Periods of continuous growth are interrupted by sudden jumps in the condensation rate that result from the coalescence events depicted in Figure 6 (a). The number of droplets used in the PSSM model predictions correspond to all the droplets enclosed in the region defined by $\kappa=2530 \mu \mathrm{m}$.

Overall, the condensation rates predicted at each instant of the growth closely match the condensation rates measured experimentally for droplets $d$ and $e$. The trends in the condensation rates of the droplet do not correspond with the ones expected from the isolated growth $\left(\dot{m} \sim t^{1 / 2}\right)$ and film-like growth ( $\dot{m}=$ constant) model [12,17]. Before the first coalesce event, the condensation rate of droplets $d$ and $e$ follow a power law $\dot{m} \sim t^{\beta}$ where $\beta=-0.11$ for droplet $d$ and $\beta=0.12$ for droplet $e$. Within an image, each droplet has distinct growth characteristics that depend on its size and its interactions with their respective neighbors, as will be explored in the subsequent sections.

\subsection{Comparison with isolated droplet and film-like models for condensation}

This section compares the experimental condensation rates of individual droplets and group of droplets, respectively presented in Sections 4.1.1 and 4.1.2, against the isolated growth and the film-like growth models. The isolated growth model given in equation (3) neglects the interaction between neighboring droplets. It assumes that droplet grow during dropwise condensation in a 
pattern such that the distance between droplet is so large that the vapor distributions surrounding each droplet have no effect on each other. In comparison with the condensation rate measured for the single droplet of interest considered in Section 4.1.1, the condensation rate predicted by the isolated model from equation (3) $\left(\dot{m}_{i s o}=6.46 \times 10^{-11} \mathrm{~kg} / \mathrm{s}\right)$ is over an order of magnitude larger. Similarly, the total condensation rate for the group of droplets considered in Section 4.1.2, predicted by the isolated model $\left(\dot{M}_{i s o}=6.42 \times 10^{-10} \mathrm{~kg} / \mathrm{s}\right)$, is two orders of magnitude larger the measured condensation rate. The isolated model for dropwise condensation significantly overpredicts experimental condensation rates.

When droplets become densely packed on a substrate, it has been commonly assumed [12, 17-19] that the water vapor distributions around each droplet overlap such that they grow as a film spread over the substrate of equivalent volume. The film-like growth model treats the vapor concentration profile as varying linearly in the direction normal to the substrate. Medici et al.[12] determined an explicit expression for the temporal evolution of the thickness of a film from which the condensation rate of each droplet is a constant given by:

$$
\dot{M}_{\text {film }}=\frac{D\left(c_{\infty}-c_{s}\right)}{\zeta} S,
$$

where $S$ is the area of the condensation substrate, and $\zeta$ is the thickness of the concentration boundary layer, which corresponds to the extent of the region where the transport by diffusion dominates over the transport due to convection. A primary challenge in applying the film-like model comes from accurately determining the value of $\zeta$. Approximate estimates of $\zeta$ are often obtained from a scaling analysis for natural convection above a horizontal cooled plate. By assuming that the transport by natural convection is negligible $(P e<1)$ in the scaling analysis, Medici et al. [12] derived an expression for $\zeta$ in terms of experimental conditions:

$$
\zeta \sim\left[\frac{D \delta^{3 / 2}}{4(\beta g \Delta T)^{1 / 2}}\right]^{1 / 3},
$$

where $\delta \sim L G r^{-1 / 5}$ is the thickness of hydrodynamic boundary layer caused by buoyancy effects, with $L$ being the characteristic length of the sample, $\Delta T=T_{\infty}-T_{\text {sub }}$, and $\beta=2 /\left(T_{\infty}+T_{\text {sub }}\right)$ is the volumetric thermal expansion coefficient for air. 
For the experiments at $R H=70 \%$, the concentration boundary layer is estimated to $\zeta \approx$ $1.0 \mathrm{~mm}$. To compare the film-like growth model to the results presented in Sections 4.1.2, the substrate area $S$ is assumed to be equal to the area of the region defined by connecting the centers of the droplets of interest (yellow line in Figure 4 (b)). From equation (6), the condensation rate predicted by the film-like growth model is equal to $\dot{M}_{\text {film }}=5.55 \times 10^{-11} \mathrm{~kg} / \mathrm{s}$, an overprediction of the measured total condensation rate for the group of droplets by $82.8 \%$.

\subsection{Intensity of droplet-to-droplet interactions}

For an arbitrary droplet $i$, the condensation correction factor $\eta$ computed from equation (4) can be rewritten as:

$$
\eta_{i}=B_{i, j=i}^{-1}+\sum_{i, j \neq i}^{N} B_{i j}^{-1}
$$

where $B_{i, j=i}^{-1}$ is greater than unity and $\sum_{i, j \neq i}^{N} B_{i j}^{-1}$ is negative and represents the contributions caused by neighboring droplets. In relation to all the droplets in the system, any given droplet $j$ reduces the condensation rate of the droplet of interest with an intensity given by:

$$
\Psi_{i j}=\frac{B_{i, j \neq i}^{-1}}{\sum_{i, j \neq i}^{N} B_{i j}^{-1}},
$$

where $\Psi_{i j}$ will be equal to unity when droplet $j$ has a dominant interaction with the droplet of interest $i$ compared to all other interacting droplets; $\Psi_{i j}$ will be equal to zero when the interaction between droplet $i$ with droplet $j$ is negligible.

Figure 7 (a) and (b) depict the absolute value of the intensity of the interaction between a central droplet of interest and all other droplets in the domain at times of $2.0 \mathrm{~min}$ and $31.0 \mathrm{~min}$, respectively. The conditions used in the calculation were as the ones used in section 4.1.3 ( $T_{\text {sub }}=$ $10{ }^{\circ} \mathrm{C}, R H=70 \%$, and $T_{\infty}=20.5{ }^{\circ} \mathrm{C}$ ). Similarly, the number of droplets included in this calculation corresponded with the droplets enclosed by a region with a maximum cord length of $\kappa=2531 \mu \mathrm{m}$. The color of each droplet represents the intensity of the interaction. The intensity of the interaction is larger for the nearest neighboring droplets and attenuates with distance from the droplet of interest. The red dashed line in the figure encloses $90 \%$ of the total interaction 
intensity between the droplet of interest and all droplets in the domain. At earlier stages of the growth within regime $(i)$, in Figure 7 (a), the sum of the interaction intensities of the nearest droplets is only $42.2 \%$ of the total interaction intensity, while the region that contains $90 \%$ of the total interaction intensity extends far beyond the nearest droplets, $\sim 1000 \mu \mathrm{m}$ from the droplet of interest. At a later time within regime (ii), in Figure 7 (b), the sum of the interaction intensities of the nearest droplets increases to $87.2 \%$ of the sum of the interaction with all neighboring droplets. In this case, the distance at which the interaction intensity sums to $90 \%$ of the total is a decreased distance comparable to the separation the nearest neighbors, $\sim 300 \mu \mathrm{m}$. Note that even though a large proportion of the total interaction intensity occurs between a droplet and its nearest neighbors, this conclusion does not mean that it is accurate to calculate the magnitude of condensation rate of a droplet by considering only its nearest neighbors (refer to Section 4.1.1). Rather, the strong interactions with the nearest neighbors governs there condensation rate relative to each other, but the interactions with all the other droplets determines the specific magnitude of the condensation rater. Models that consider only the interactions with nearest droplets offer a limited description of droplet growth that can predict trends, but not quantitative condensation rates.

\subsection{Effects of droplet size and spatial distribution on the condensation rate}

This section focuses on using the predictions of the point sink superposition method to gain insight into the effects of droplet size and spatial distribution on the condensation rate of each droplet within the system. Figure 8 (a) shows individual droplet condensation rates versus their corresponding contact radius for a set of images taken at $t=2.0,6.0,21.0$ and $31.0 \mathrm{~min}$. At any given time, the condensation rate is proportional to droplet size. As time proceeds, the distribution

of droplet sizes broadens and the average droplet size increases; as a result, the average condensation rate and the range of condensation rates observed in each image increases with time. However, across different times, larger droplets do not always necessarily grow at larger rates; droplets having similar sizes but belonging to images taken at different times can have significantly different condensation rates. For example, droplets ranging from a contact radius of $\sim 40 \mu \mathrm{m}$ to $\sim 45 \mu \mathrm{m}$ at $t=6.0 \mathrm{~min}$ grow at higher rates than the same size droplets at $21.0 \mathrm{~min}$. This behavior could not be captured by dropwise condensation models that calculate the total condensation rate by summing the condensation rates of individual droplets as they were isolated from one other. 
These differences in the condensation rates of droplets can be explained in terms of the spatial distribution of droplets in their surroundings and their size relative to the size of their neighbors. Voronoi polygon constructions based on the droplet center positions have been used extensively to characterize condensation rates and relative distance from neighboring droplets. Prior studies $[12,17,20-23]$ have suggested that droplets with larger Voronoi polygons grow at faster rates; the area of the Voronoi polygon is the sum of the footprint area of the droplet located at the center of the polygon and the bare area surrounding the droplet. Large droplets that are surrounded by large bare areas are able to capture relatively more of the water vapor that is diffusing from the ambient toward the substrate and laterally in between neighboring droplets. Figure 9 (a) and (b) shows the predicted condensation rates and Voronoi constructions of droplets from snapshot images taken at $t=2 \mathrm{~min}$ and $t=31 \mathrm{~min}$, respectively. The color of each droplet indicates its condensation rate. The red lines define the boundary of each Voronoi polygon, and the color inside each polygon represents the magnitude of the polygon area. At $t=2 \mathrm{~min}$, the droplets that grow at faster rates have polygons with larger areas even though they have similar size, such as comparing droplet $f$ to droplet $g$ in Figure 8 (b). At later times, droplets that have polygons with larger areas do not necessarily grow faster. We have previously shown that the condensation rate of pairs of droplets predicted by the PSSM is sensitive to the ratio of between the droplet sizes [15]. Large droplets can limit the vapor diffusion to small droplets in their surroundings For example, droplet $h$ in Figure 9 (b) has a larger Voronoi polygon area than its neighbor droplet $l$, but grows at a smaller rate, because droplet $l$ has a larger size and it is surrounded by smaller neighbors than droplet $h$. Thus, the size of a given droplet in relation to the size of its neighbors can significantly influence the condensation rate.

Figure 8 (b) shows individual droplet condensation rates versus their corresponding ratio between the contact radius of each droplet to the average contact radius of its nearest neighbors $R_{c i} /\left\langle R_{c j}\right\rangle$ for a set of images taken at $t=2.0,6.0,21.0$ and $31.0 \mathrm{~min}$. The condensation rate of individual droplets increases with the ratio between their contact radii and the average contact radius of their nearest neighbors. At the earliest stages of the growth, when the droplets have similar sizes as shown in Figure 8 (a), the condensation rate is relatively insensitive to the droplet relative size and the condensation rate primarily $\operatorname{dep} R_{c i} /\left\langle R_{c j}\right\rangle$ ends on the spatial distribution of 
the droplets. At later times, similar variations in droplet relative size lead to greater changes in the condensation rate.

\subsection{Conclusions}

The point source superposition method is used to predict experimental condensation rates of individual droplets, within a system of many droplets on a substrate, formed during vapordiffusion-driven dropwise condensation from humid air. The point sink superposition method accurately predicts the experimental growth rates of individual droplets, and the total condensation rate of groups of interacting droplets, by superposing the vapor distributions of all droplets on the substrate. Assuming that droplets grow completely isolated from one another severely overpredicts the measured condensation rates. It is shown through use of the point sink superposition method that a large number of interacting droplets, over a length scale of the substrate much larger than the separation distance between droplets, must be considered to accurately predict the experiments. Even though the interaction between nearest neighboring droplets is the strongest, only including the such neighboring droplets in the close vicinity still severely overpredicts condensation rate of an individual within the system. This highlights a limitation of modeling approaches that describe the condensation rate based on the interactions between nearest neighbors. The point superposition method, which captures the interactions between all droplets within the system and accounts for their locations and sizes, is used as a tool study the interactions between condensing droplets in the experiments as a function of droplet size and pitch. Relative droplet size effects on condensation rates are more prominent at later stages of the growth, when the size distribution of droplet is broader.

\section{Acknowledgments}

The first author acknowledges the financial support provided by the Colombian Department

for Science, Technology and Innovation (Colciencias) and the Colombia-Purdue Institute (CPI). Special thanks to Prof. Suresh V. Garimella in the School of Mechanical Engineering at Purdue University for technical discussions related to this work. 


\section{References}

[1] M. Tomaszkiewicz, M. Abou Najm, D. Beysens, I. Alameddine, and M. El-Fadel, "Dew as a sustainable non-conventional water resource: a critical review," Environmental Reviews.,23 (4), (2015), 425-442.

[2] H. Vuollekoski, M. Vogt, V.A. Sinclair, J. Duplissy, H. Järvinen, E.M. Kyrö, R. Makkonen, T. Petäjä, N.L. Prisle, P. Räisänen, M. Sipilä, "Estimates of global dew collection potential on artificial surfaces", Hydrology Earth System Sciences, 19 (1), (2015), 601-613.

[3] D. Beysens, M. Muselli, I. Milimouk, C. Ohayon, S.M. Berkowicz, E. Soyeux, M. Mileta, P. Ortega, "Application of passive radiative cooling for dew condensation", Energy, 31 (13) (2006), 2303-2315.

[4] B. Gido, E. Friedler, and D. M. Broday, "Assessment of atmospheric moisture harvesting by direct cooling", Atmospheric Research, 182, (2016), 156-162.

[5] A. Lee, M.-W. Moon, H. Lim, W.-D. Kim, and H.-Y. Kim, "Water harvest via dewing", Langmuir, 28 (27), (2012), 10183-10191.

[6] A. Ghosh, S. Beaini, B. J. Zhang, R. Ganguly, and C. M. Megaridis, "Enhancing dropwise condensation through bioinspired wettability patterning”, Langmuir, 30 (43),(2014), $13103-13115$.

[7] X. Chen, J. A. Weibel, and S. V. Garimella, "Exploiting microscale roughness on hierarchical superhydrophobic copper surfaces for enhanced dropwise condensation", Advanced Materials Interfaces, 2 (3), (2015)1400480

[8] K.-C. Park, Kim, P., Grinthal, A., He, N., Fox, D., Weaver, J. C., and Aizenberg, J.., "Condensation on slippery asymmetric bumps", Nature, 531 (7592), (2016), 78-82.

[9] D. Milani, A. Abbas, A. Vassallo, M. Chiesa, and D. A. Bakri, "Evaluation of using thermoelectric coolers in a dehumidification system to generate freshwater from ambient air", Chemical Engineering Science, 66 (12), (2011), 2491-2501.

[10] I. O. Ucar and H. Y. Erbil, "Use of diffusion controlled drop evaporation equations for dropwise condensation during dew formation and effect of neighboring droplets", Colloids and Surfaces A: Physicochemical and Engineering Aspects, 411, (2012), 60-68.

[11] J. E. Castillo, J. A. Weibel, and S. V. Garimella, "The effect of relative humidity on dropwise condensation dynamics", International Journal of Heat and Mass Transfer, 80, (2015), 759-766.

[12] M.-G. Medici, A. Mongruel, L. Royon, and D. Beysens, "Edge effects on water droplet condensation," Physical Reviews E, 90 (6), (2014), 062403.

[13] S. Nath and J. B. Boreyko, "On localized vapor pressure gradients governing condensation and frost phenomena", Langmuir, 32 (33), (2016), 8350-8365.

[14] S. Nath, S. F. Ahmadi, and J. B. Boreyko, "A review of condensation frosting", Nanoscale and Microscale Thermophysical Engineering, 21(2), (2017), 81-101.

[15] J. E. Castillo and J. A. Weibel, "A point sink superposition method for predicting droplet interaction effects during vapor-diffusion-driven dropwise condensation in humid air", International Journal of Heat and Mass Transfer, 118, (2018), 708-719.

[16] Y. O. Popov, "Evaporative deposition patterns: Spatial dimensions of the deposit", Physical Review E, 71 (3), (2005), 036313.

[17] M. Sokuler, G. K. Auernhammer, C. J. Liu, E. Bonaccurso, and H.-J. Butt, "Dynamics of Condensation and Evaporation: Effect of Inter-Drop Spacing," European Physics Letters, 89 (3), (2010), 36004. 
[18] B. J. Briscoe and K. P. Galvin, "Growth with coalescence during condensation", Phys. Rev. A, 43 (4), (1991), 1906-1917.

[19] D. Beysens, "Dew nucleation and growth", Comptes Rendus Physique, 7 (9-10), (2006), 1082-1100..

[20] J. Guadarrama-Cetina, R. D. Narhe, D. A. Beysens, and W. González-Viñas, "Droplet pattern and condensation gradient around a humidity sink", Physical Review E, 89 (1), (2014), 012402.

[21] A. Steyer, P. Guenoun, D. Beysens, and C. M. Knobler, "Two-dimensional ordering during droplet growth on a liquid surface”, Physical Review B, 42 (1), (1990), 1086-1089.

[22] P. Meakin, "Dropwise condensation: the deposition growth and coalescence of fluid droplets," Physica Scripta., T44, (1992), 31.

[23] J. Blaschke, T. Lapp, B. Hof, and J. Vollmer, "Breath figures: Nucleation, growth, coalescence, and the size distribution of droplets", Physical Review Letters, 109 (6), (2012).

[24] J. L. Viovy, "Scaling description for the growth of condensation patterns on surfaces", Physical Review A, 37 (12), (1988), 4965-4970 
Figures

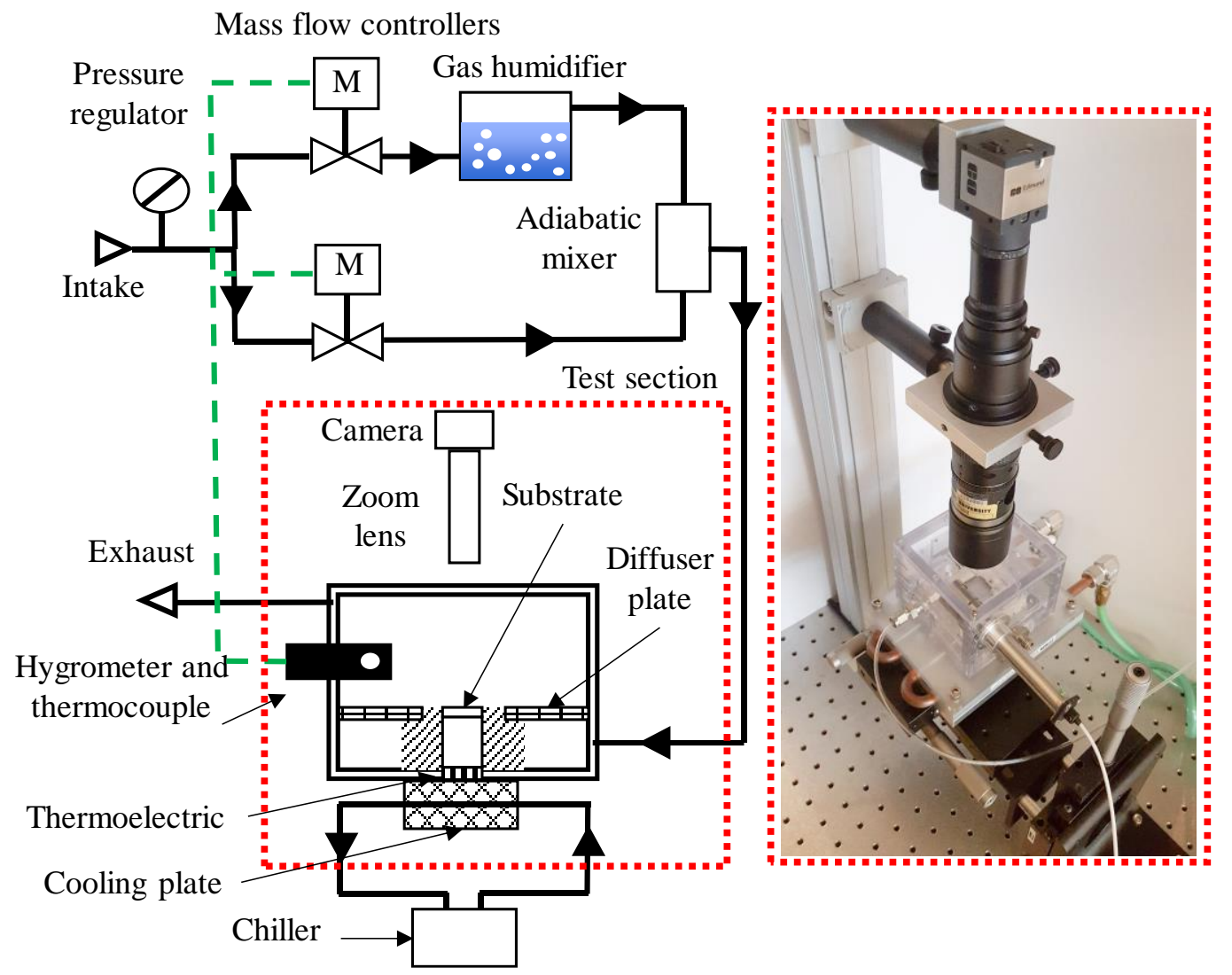

Figure 1. (a) Schematic diagram depicting the condensation testing faculty and a photograph of the test section.

Note for the editor: 1.5 column figure 


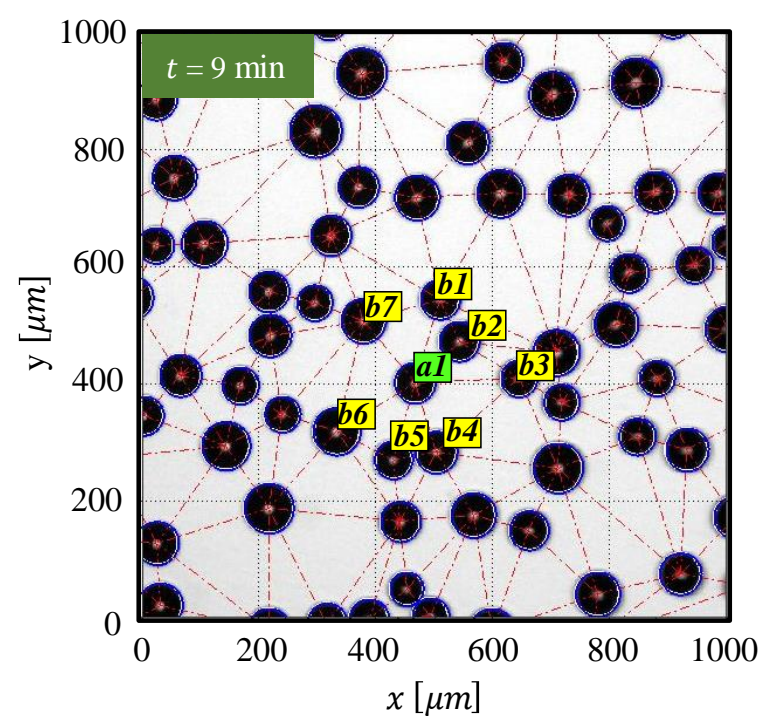

(a)

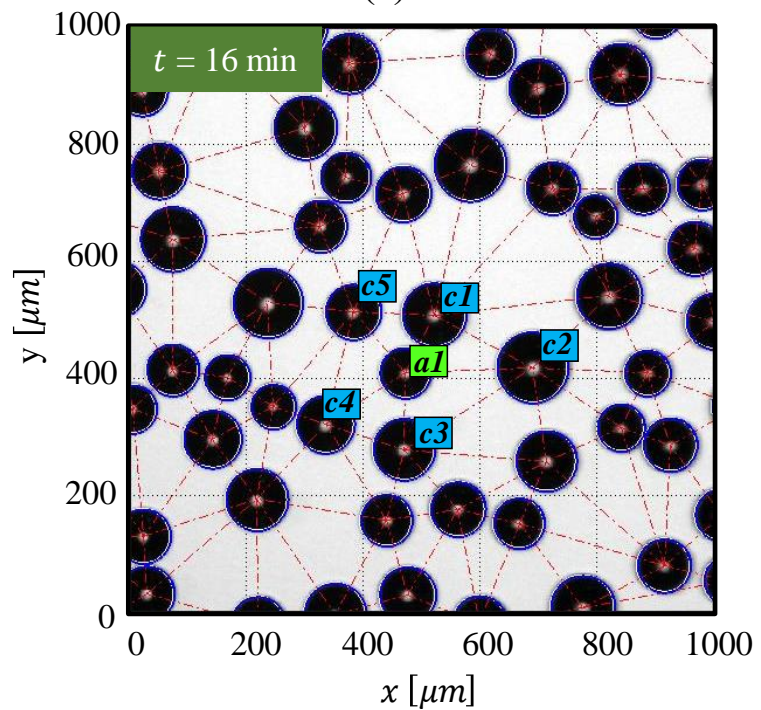

(b)

Figure 2. Example snapshot images at (a) 9 min to (b) 16 min following droplet recognition and tracking. $\left(T_{\text {sub }}=10^{\circ} \mathrm{C}, R H=70 \%\right.$, and $\left.T_{\infty}=20.5^{\circ} \mathrm{C}\right)$

Note for the editor: Single column figure 


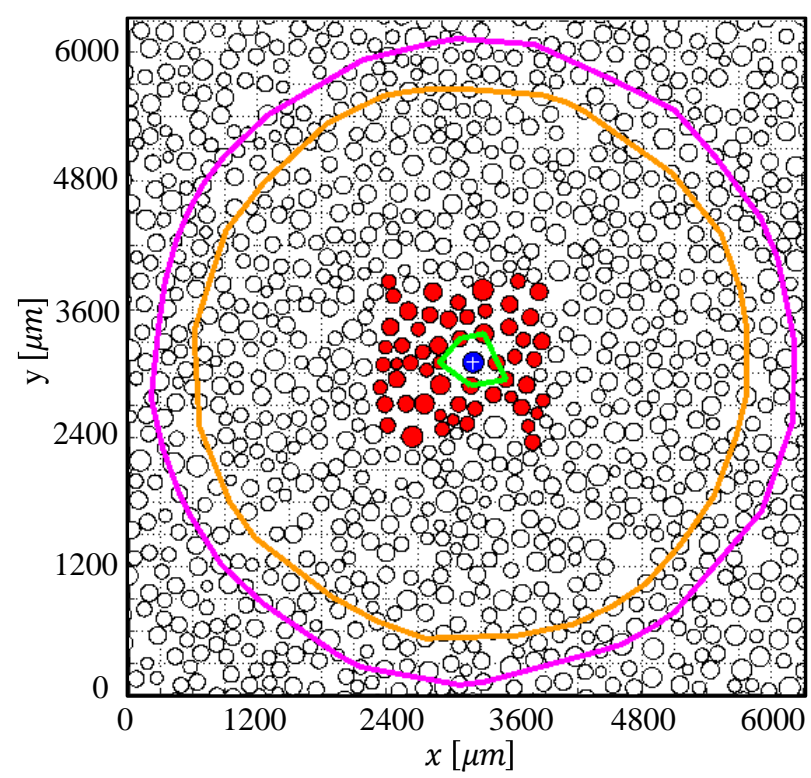

(a)

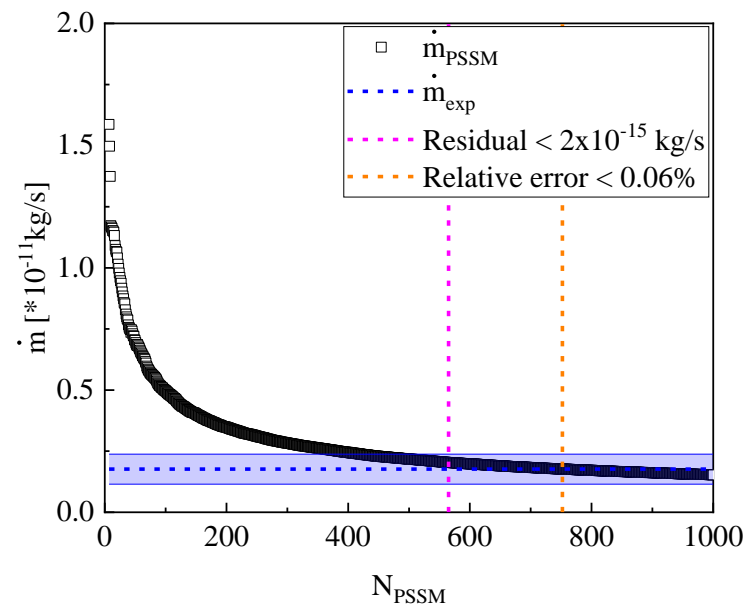

(b)

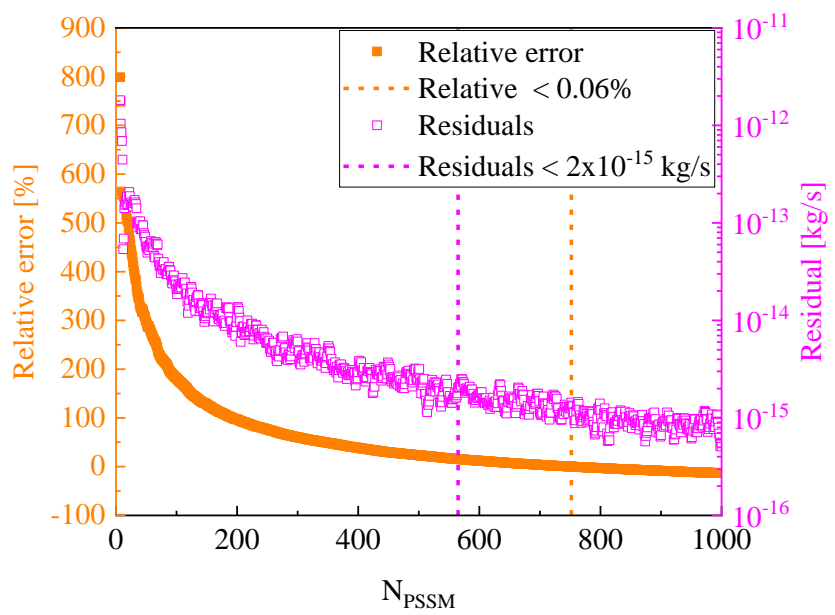

(c) 
Figure 3. Prediction of the condensation rate of a single droplet within a system of many droplets $\left(T_{\text {sub }}=10{ }^{\circ} \mathrm{C}, R H=70 \%, T_{\infty}=20.5^{\circ} \mathrm{C}\right.$ ). (a) Top-down view of the domain used for the PSSM calculation, consisting of droplets inside the experimental field of view at $t=31 \mathrm{~min}$ (filled red) and artificial droplets generated outside the field of view (filled white). (b) Predicted condensation rate for the droplet of interest, filled blue in (a), as the number of neighboring droplets considered in the model increases. The blue shaded region defines upper and lower bounds of the experimental error. (c) Relative error between the predicted and measured condensation rate and residual of the condensation rate between increments in the number of droplets included. The purple and orange rings in (a) encompass all droplets necessary to predict $\dot{m}_{i s o}$ with a residual $<2 \times 10^{-15} \mathrm{~kg} / \mathrm{s}$ and relative error $0.06 \%$ (also shown with corresponding dashed vertical lines in (b) and (c)).

Note for the editor: Single column figure 


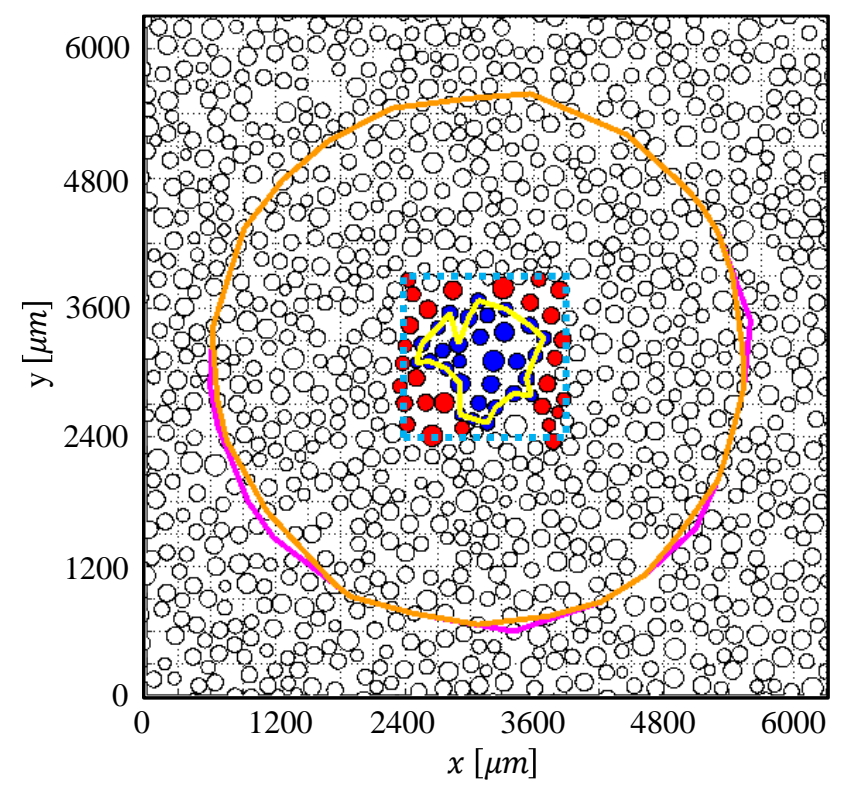

(a)

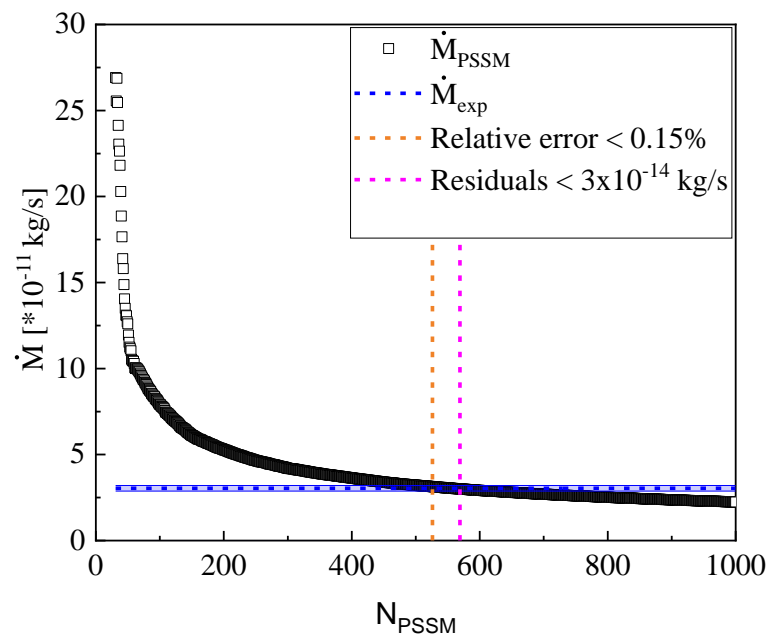

(b)

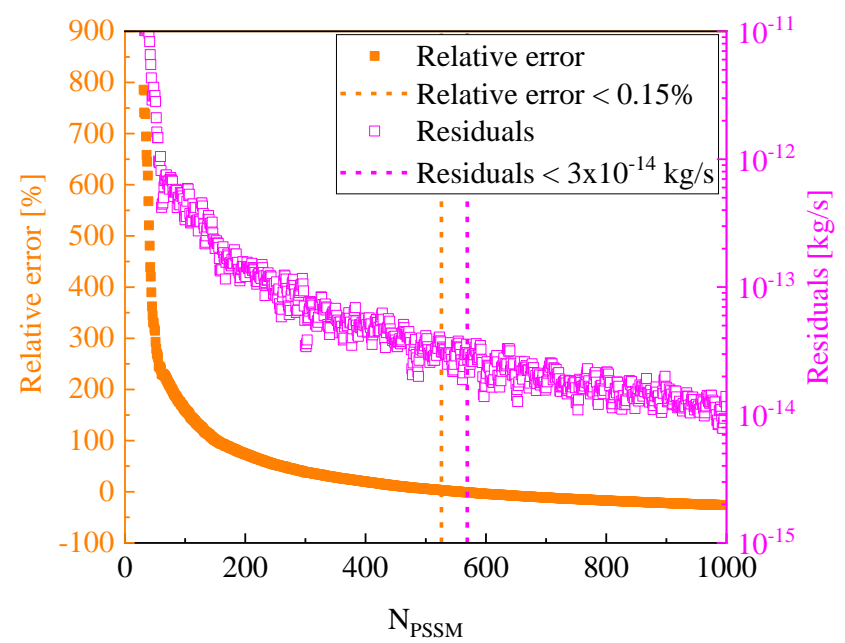

(c) 
Figure 4. Prediction of the total condensation rate for 30 droplets within a system of many droplets $\left(T_{\text {sub }}=10^{\circ} \mathrm{C}, R H=70 \%, T_{\infty}=20.5^{\circ} \mathrm{C}\right.$ ). (a) Top-down view of the domain used in the PSSM calculation with droplets inside the field of view at $t=31 \mathrm{~min}$ (filled red) and artificial droplets generated outside the field of view (filled white). (b) Predicted total condensation rate for the group of droplets filled blue in (a) versus the number of neighboring droplets considered in the model. The blue shaded region defines upper and lower bounds of the experimental error. (c) Relative error between the predicted and measured total condensation rate and the residual of the total condensation rate between increments in the number of droplets included. The purple and orange rings in (a) encompass all droplets necessary to predict $\dot{m}_{\text {iso }}$ with a residual $<3 \times 10^{-14} \mathrm{~kg} / \mathrm{s}$ and relative error $0.15 \%$ (also shown with corresponding dashed vertical lines in (b) and (c))

Note for the editor: Single column figure 


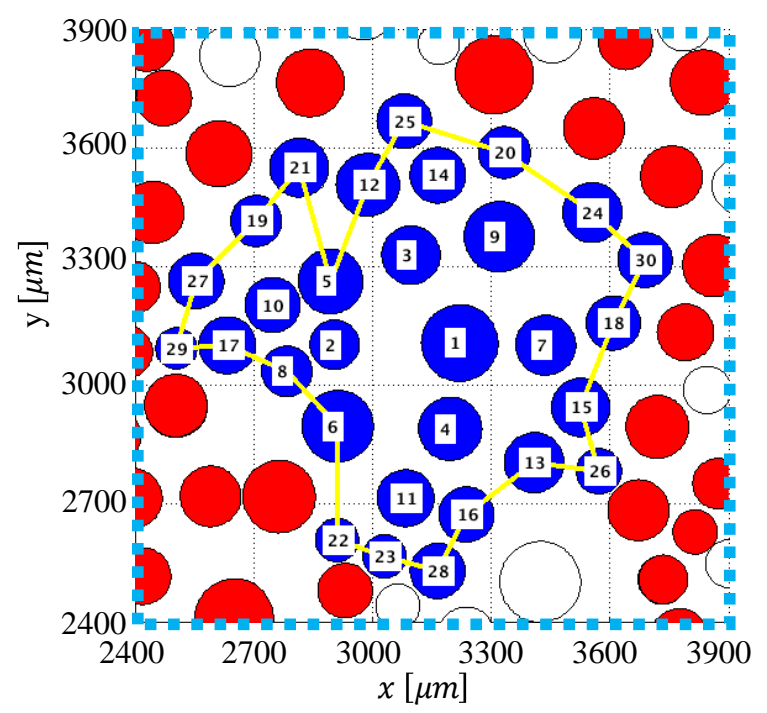

(a)

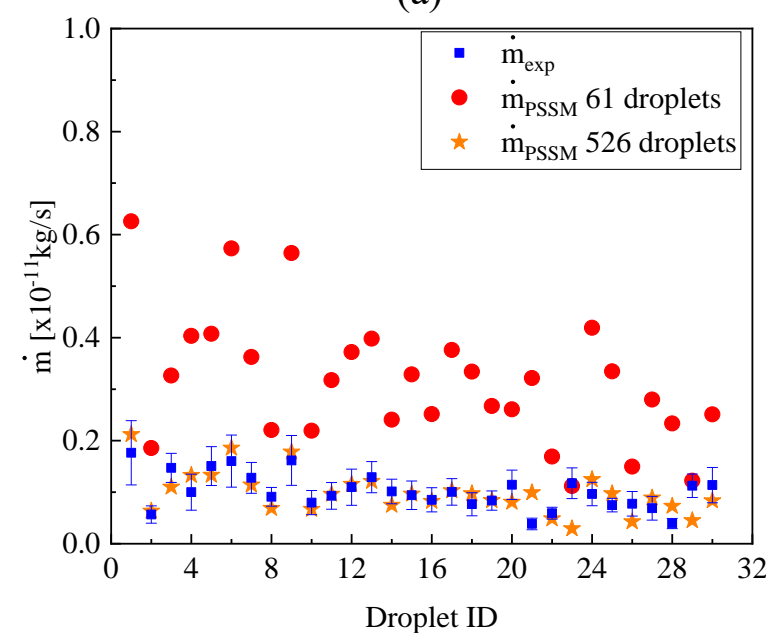

(b)

Figure 5. Prediction of individual condensation rate of a group of 30 droplets within a system of many droplets $\left(T_{\text {sub }}=10^{\circ} \mathrm{C}, R H=70 \%, T_{\infty}=20.5^{\circ} \mathrm{C}\right)$. (a) Magnified view of Figure 4 (a) showing the field of view and including tags on the droplets of interest. (b) Measured and predicted individual condensation rates for each tagged droplet of interest.

Note for the editor: Single column figure 


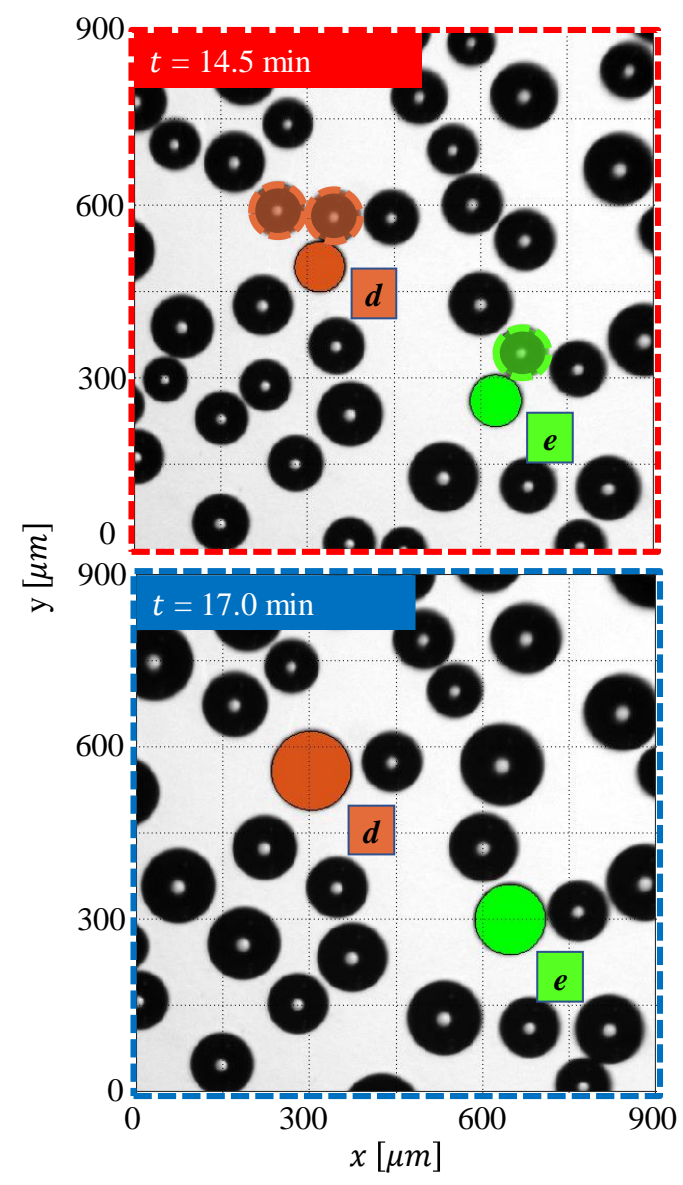

(a)

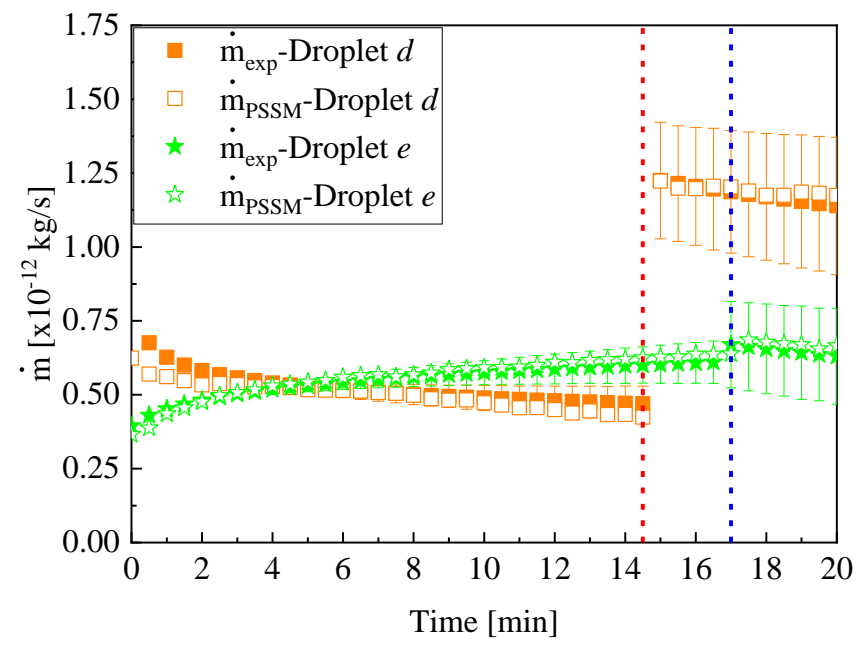

(b)

Figure 6. (a) Snapshot images of droplets condensing during condensation experiments at $T_{\text {sub }}=$ $10{ }^{\circ} \mathrm{C}, R H=70 \%, T_{\infty}=20.5^{\circ} \mathrm{C}$. The sequence of images shows droplets $d$ and $e$ before $(t=$ 
$14.5 \mathrm{~min}$ ) and after (and $t=17.0 \mathrm{~min}$ ) their respective first coalescence events with neighboring droplets. (b) Temporal evolution of the condensation rates of droplets $d$ and $e$ with vertical lines indicating times that corresponds with the images in (a).

Note for the editor: Single column figure 


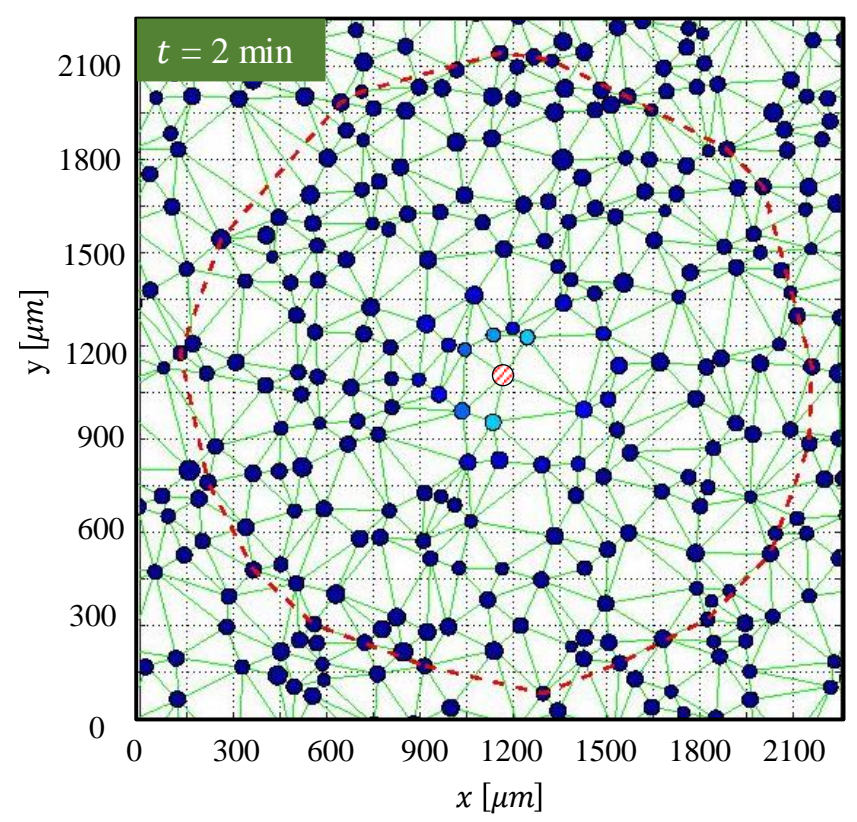

(a)

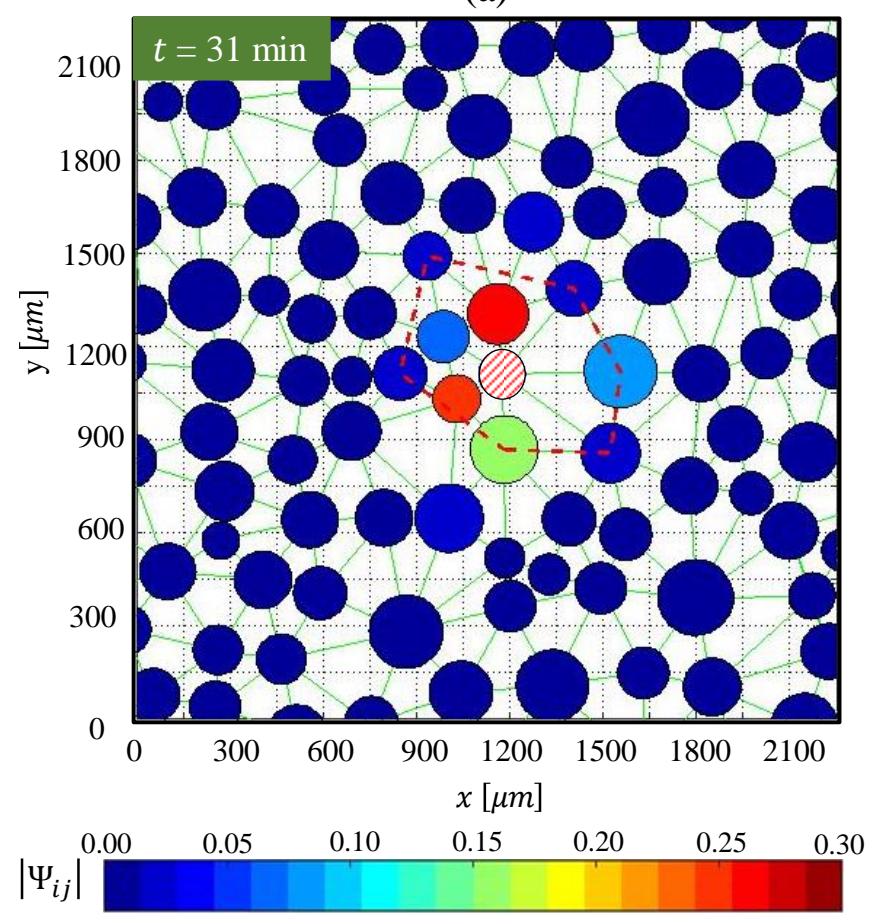

(b)

Figure 7. Absolute value of the intensity of interaction between the droplet of interest (dashed circle near the center) with all other droplets in the domain, calculated based on the images taken at (a) $t=2 \mathrm{~min}$ and (b) $t=31 \mathrm{~min}$. The fill color of the droplets corresponds to the interaction intensity contour scale. $\left(T_{\text {sub }}=10^{\circ} \mathrm{C}, R H=70 \%, T_{\infty}=20.5^{\circ} \mathrm{C}\right)$

Note for the editor: Single column figure 


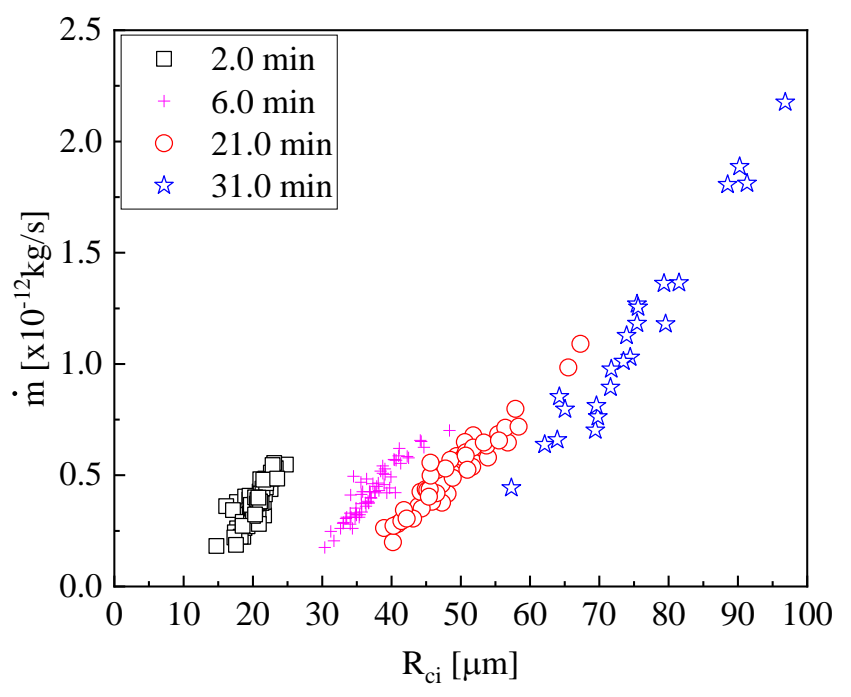

(a)

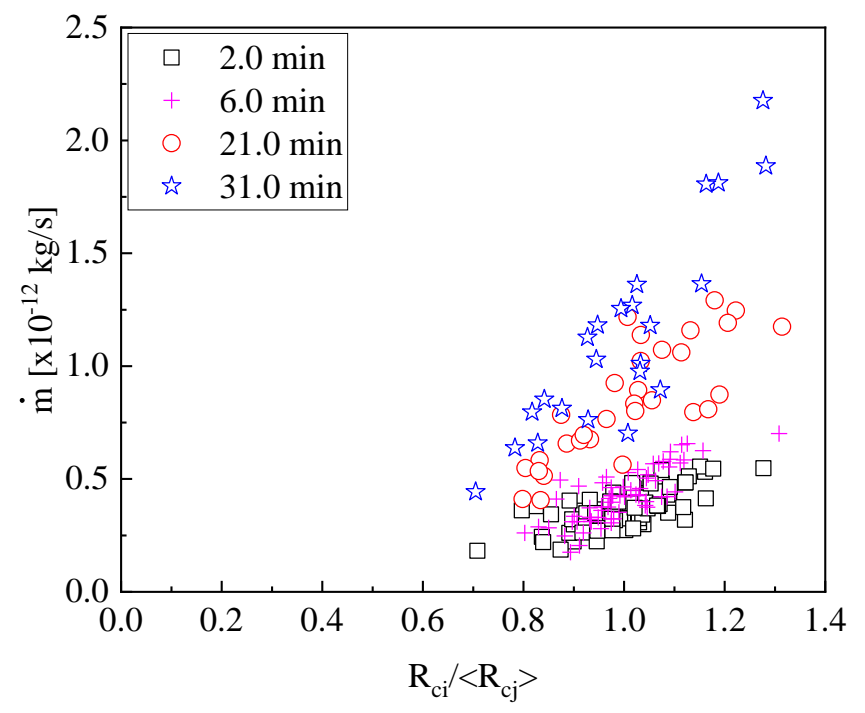

(b)

Figure 8. Condensation rate of each droplet as a function of (a) their respective contact radius and (b) the ratio between the contact radius of each droplet and the average contact radius of its nearest neighbors. Results are shown for the experimental conditions $T_{\text {sub }}=10^{\circ} \mathrm{C}, R H=70 \%$, and $T_{\infty}=$ $20.5^{\circ} \mathrm{C}$ at $t=2,6,21$ and $31 \mathrm{~min}$.

Note for the editor: Single column figure 


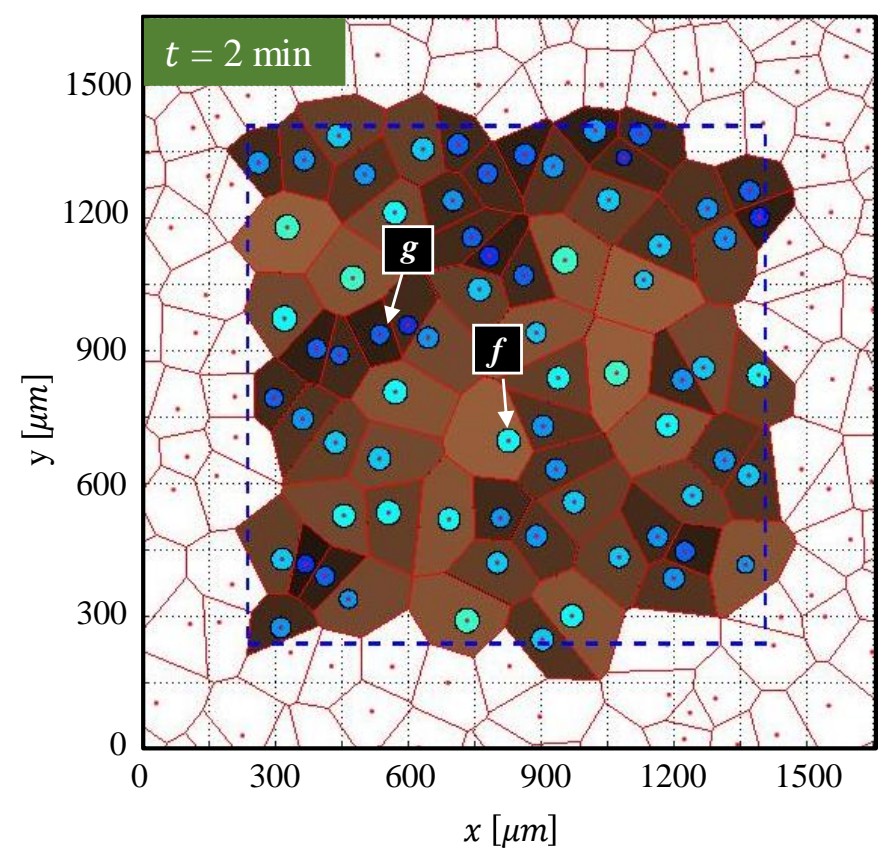

(a)
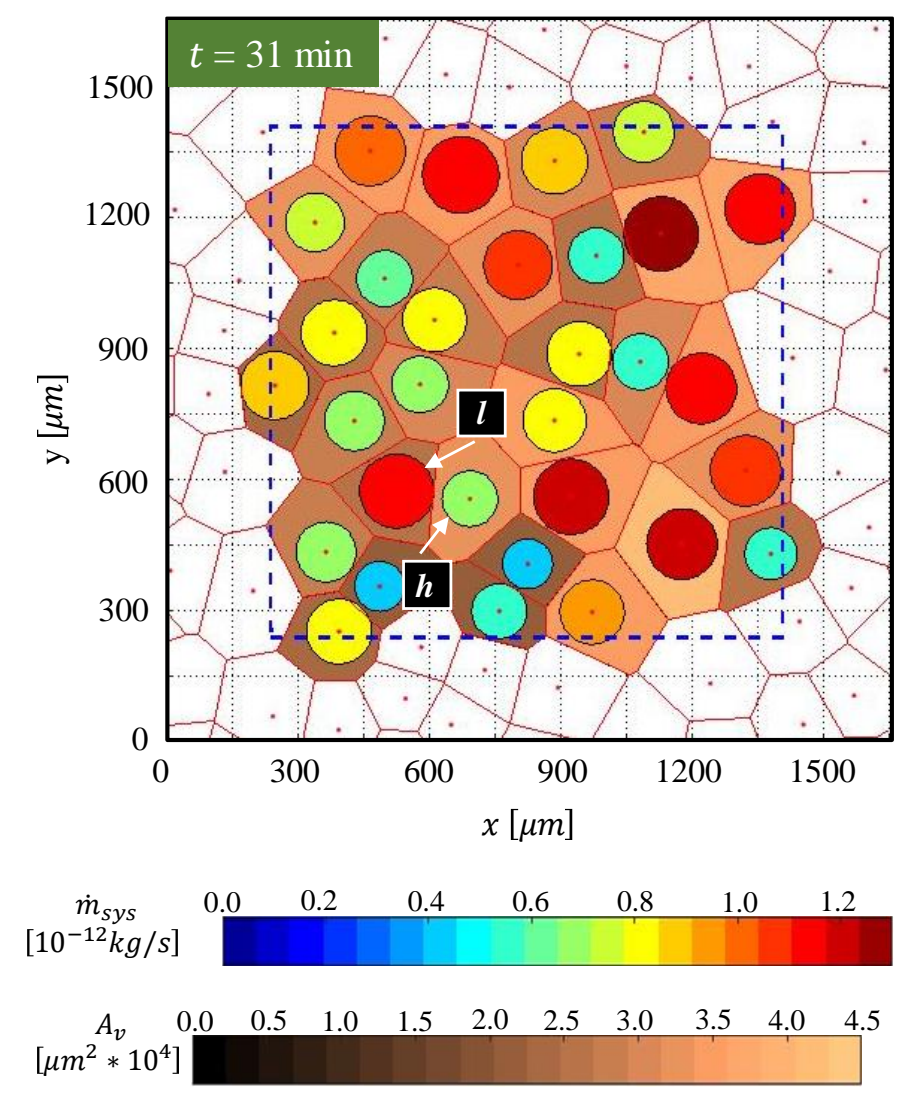

(b) 
Figure 9. Condensation rates and Voronoi polygons for droplets in the model domain corresponding to images at (a) $t=2$ and (b) $t=31 \mathrm{~min}\left(T_{\text {sub }}=10^{\circ} \mathrm{C}, R H=70 \%\right.$, and $T_{\infty}=20.5$ ${ }^{\circ} \mathrm{C}$ ). The color filling each circle indicates the predicted condensation rate of the droplet, while the color of the Voronoi polygons around each droplet represents the magnitude of the polygon area.

Note for the editor: Single column figure 


\section{Tables}

Table 1. Maximum cord length of the region ( $\kappa$ ) that encloses the given number of droplets ( $N_{P S S M}$ ) required to predict the measured condensation rate with minimum relative error (for a group of 30 droplets at different times during of experiment at $T_{\text {sub }}=10^{\circ} \mathrm{C}, R H=70 \%$, and $T_{\infty}=$ $\left.20.5^{\circ} \mathrm{C}\right)$.

\begin{tabular}{ccc}
\hline $\begin{array}{c}\text { Time } \\
{[\mathbf{m i n}]}\end{array}$ & $\boldsymbol{N}_{P S S M}$ & $\boldsymbol{\kappa}[\boldsymbol{\mu m}]$ \\
\hline 11 & 982 & 2330 \\
16 & 820 & 2340 \\
21 & 811 & 2790 \\
26 & 586 & 2600 \\
31 & 526 & 2620 \\
\hline
\end{tabular}

